\title{
Progress, challenges and new concepts in microRNAs
}

\author{
ZHANG YouYi \\ Institute of Vascular Medicine, Peking University Third Hospital, Key Laboratory of Cardiovascular Molecular Biology and Regulatory \\ Peptides, Ministry of Health and Key Laboratory of Molecular Cardiovascular Sciences, Ministry of Education, Beijing 100191, China
}

Received November 15, 2011

Citation: ZHANG Y Y. Progress, challenges and new concepts in microRNAs. Sci China Life Sci, 2011, 54: 1096, doi: 10.1007/s11427-011-4259-6

MicroRNAs are a group of small noncoding RNAs. They have rapidly gained attention in the field as novel regulators of cellular morphology and function. Currently hundreds of microRNAs have been described in human genome. MicroRNAs play important roles in posttranscriptional regulation of gene expression by inhibiting protein translation and/or promoting mRNA degradation. MicroRNAs were initially thought to be subtle regulators of gene expression, but increasing evidence demonstrates that the regulatory functions of microRNAs are crucial for the cell. MicroRNAs have been found to be involved in the development, tissue homeostasis as well as diseases. However, at present, the pathological implications of deregulated microRNA expression in diseases remain largely unknown. In these reviews, the authors focus on recent advancement on regulation of microRNA biogenesis and function in cardiac and neuronal development and disease. Zhang Y et al. summarized the mechanism of electrical remodeling, structural remodeling and intracel- lular calcium handling remodeling in atrial fibrillation and the association of microRNAs network with them. Feng Y L et al. illustrated the current insights into the regulation of microRNAs in heart development and diseases, such as cardiac hypertrophy, ischemic cardiomyopathy and arrhythmia. Feng W et al. focused on microRNA biogenesis and function in neuronal and glial development in the mammalian brain, and dysregulation of the microRNA pathway in neurodevelopmental and neurodegenerative diseases. The value to readers is that three authors introduced the results studied by their groups in the reviews. The functions of microRNAs were better interpreted. Also they discussed that the potential perspective of microRNAs expression pattern serves as informative biomarkers that indicate the functional status as well as progression and prognosis in diseases. Based on the fast growing knowledge for microRNAs, they speculate a promising translational future for microRNA diagnostics and therapeutics.

Open Access This article is distributed under the terms of the Creative Commons Attribution License which permits any use, distribution, and reproduction in any medium, provided the original author(s) and source are credited.

email: zhangyy@bjmu.edu.cn 